Org Lett. 2019 October 04; 21(19): 8027-8030. doi:10.1021/acs.orglett.9b03015.

\title{
Synthesis of the EFG Framework of Tamulamides A and B
}

\author{
Elizabeth H. Kelley ${ }^{\dagger}$, Timothy F. Jamison ${ }^{*}$ \\ "Department of Chemistry, Massachusetts Institute of Technology, 77 Massachusetts Avenue, \\ Cambridge, Massachusetts, 02139, United States
}

\begin{abstract}
Synthesis of the fused polycyclic ether motif comprising the $E F G$ rings of the marine ladder polyethers tamulamides A and B has been achieved via two different etherification strategies. Ultimately, a reductive etherification approach proved most successful due to tolerance of the $G$ ring substitution and provided the $E F G 6,7,6$ ring system in $58 \%$ yield.
\end{abstract}

\section{Graphical Abstract}

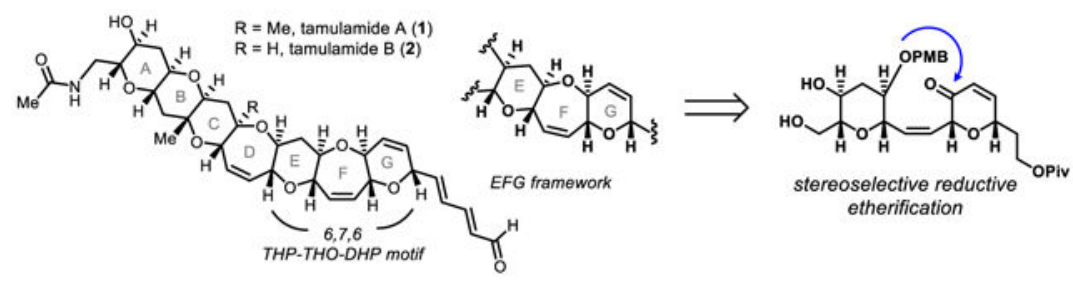

Tamulamides A (1) and B (2) are two members of the marine ladder polyether (MLP) family of natural products (Figure 1a). ${ }^{1}$ Isolated in 2010, the two tamulamides contain seven fused rings and 15 stereogenic centers and their sole difference is the substituent on the central $D$ ring (tamulamide $\mathrm{A}, \mathrm{R}=\mathrm{Me}$; tamulamide $\mathrm{B}, \mathrm{R}=\mathrm{H}$ ). ${ }^{2}$ Binding at the same voltage sensitive sodium channels as brevenal, (tamulamide $\mathrm{A}, \mathrm{K}_{\mathrm{i}}=2.5 \mu \mathrm{M}$; tamulamide $\mathrm{B}, \mathrm{K}_{\mathrm{i}}=210 \mathrm{nM}$ ), the tamulamides appear to also possess brevenal's purported antagonistic activity, as they exhibit no significant ichthyotoxicity at any level tested. ${ }^{2}$ The biological importance of the MLP family of natural products is well established, with many polyethers possessing antifungal, ${ }^{3}$ anticancer ${ }^{4}$ and antibiotic ${ }^{5}$ activity.

Inspiration for the synthesis of MLPs has been drawn from their proposed biosynthesis, in which the entire polyether framework is formed from an epoxide opening cascade of a polyepoxide precursor. ${ }^{6}$ This proposed synthesis, while certainly elegant, poses significant challenges when the regiose-lectivity of the epoxide-opening event is taken into account. Cyclization of electronically-unbiased epoxides occurs preferentially at the exo rather than

\footnotetext{
"Corresponding Author tfj@mit.edu.

$\dagger$ Present Addresses Relay Therapeutics, 399 Binney Street, $2^{\text {nd }}$ Floor, Cambridge, MA, USA, 02139

Supporting Information

The Supporting Information is available free of charge on the ACS Publications website.

Experimental procedures and ${ }^{1} \mathrm{H}$ and ${ }^{13} \mathrm{C}$ spectral data for compounds 5-12, 14-21, and X-Ray crystallographic data for $\left.12 \mathrm{PDF}\right)$.

The authors declare no competing financial interest.
} 
the endo position (Figure 1b). This results in formation of the undesired, smaller-ring THF products under acidic, ${ }^{7}$ basic ${ }^{8}$ and neutral conditions. ${ }^{9}$ Methodology developed in our group illustrated the utility of templating, where a preformed THP or 1,3 dioxane ring can bias for 6-endo over 5-exo epoxide openings. ${ }^{10,11}$ Additionally, under neutral water conditions, exclusive formation of the 6-exo opened THP product is observed rather than the oxepine that would result from 7 -endo opening. ${ }^{12}$

Two main etherification approaches to the $E F G$ rings of the tamulamides were pursued. As illustrated in Scheme 1a, this tricyclic portion of the molecule is composed of a 6,7,6tetrahydropyran-tetrahydrooxepine(THO)-dihydropyran (DHP) ring system with 7 stereogenic centers and a dienal side-chain. To the best of our knowledge, construction of such a 6,7,6 motif with a THO-DHP ring fusion has not been described in the literature. ${ }^{13}$ Historically, seven-membered rings have been difficult to access ${ }^{14}$ and in the polyether literature, approaches towards these rings utilize ring-closing metathesis ${ }^{15}$ or ring expansion methodologies. ${ }^{16}$ Access to seven membered rings containing methyl substitution at the ring junctions is well precedented both within our group ${ }^{17}$ and in the MLP field. ${ }^{18}$ However, these electrophilic cascades are not successful in the absence of the methyl directing groups, and thus do not provide access to unsubstituted ring systems such as contained within the $E F G$ motif of the tamulamides. Herein we describe the successful approaches towards this $6,7,6$ ring system via selective activation and intramolecular etherification of $\mathbf{3}$ and reductive etherification of precursor 4 (Figure 1c).

Our first etherification strategy towards the $E F G$ core began by exploring conditions to form the $F$ ring via intramolecular closure of diol $\mathbf{5}$. This diol was accessed in 13 steps (LLS) via Wittig coupling of aldehyde $\mathbf{6}$ and phosphonium salt $\mathbf{7}$ (Scheme 1). These coupling partners were synthesized from commercially available 2-deoxy-D-ribose and tri-O-acetyl-Dgalactal, respectively. Aldehyde 6 was accessed via ozonolysis of known alkene 8. ${ }^{19}$ Synthesis of the triphenyl phosphonium salt $\mathbf{7}$ began via Ferrier addition of a hydride nucleophile to tri- $O$-acetyl- $D$-galactal (Scheme 1b). Subsequent deacylation, bis TBS protection followed by selective primary TBS deprotection provided alcohol $9 .{ }^{20}$ Tosylation of the primary alcohol followed by a Finkelstein reaction ${ }^{21}$ furnished the primary iodide which was reacted with molten triphenylphosphine ${ }^{22}$ to furnish the required coupling partner 7. The Wittig coupling proceeded in good yield to furnish 10 with excellent $Z$ :E selectivity. Essential to the efficiency of this transformation was rigorous drying of the phosphonium salt $\mathbf{7}$ before its subjection to basic conditions, as was observed in the literature for similar Wittig transformations. ${ }^{23}$ Finally, desilylation furnished the intramolecular cyclization precursor, diol 5.

Reagents and conditions: a) $\mathrm{BF}_{3} \cdot \mathrm{OEt}_{2}, \mathrm{Et}_{3} \mathrm{SiH}, \mathrm{K}_{2} \mathrm{CO}_{3}, \mathrm{MeOH}, 78 \%$ over 2 steps; b) TBSCl, imid., DMF, 58\%; c) p-TsOH, MeOH, $0{ }^{\circ} \mathrm{C}, 70 \%$; d) TsCl, $1: 2$ pyr. $/ \mathrm{CH}_{2} \mathrm{Cl}_{2}, 92 \%$; e) NaI, 2-butanone, $80{ }^{\circ} \mathrm{C}, 44 \%, 74 \%$ brsm; f) $\mathrm{PPh}_{3}, 110{ }^{\circ} \mathrm{C}$, quant. g) $\mathrm{O}_{3}, \mathrm{PPh}_{3}, \mathrm{MeOH}, 85 \%$; h) NaHMDS, then 6, 3:1 THF: HMPA, $-78{ }^{\circ} \mathrm{C}, 73 \%$, >95:5 Z/E; i) TBAF, THF, $81 \%$; j) $\mathrm{Ms}_{2} \mathrm{O}, 2$, 6-lutidine, $\mathrm{CH}_{2} \mathrm{Cl}_{2}, 0{ }^{\circ} \mathrm{C}$, quant.; k) $\mathrm{Ms}_{2} \mathrm{O}, 2$, 6-lutidine, $\mathrm{CH}_{2} \mathrm{Cl}_{2}, 0{ }^{\circ} \mathrm{C}$ to rt, $24 \mathrm{~h}$; $60{ }^{\circ} \mathrm{C} 3 \mathrm{~d}, 7 \%$ over 2 steps. DMF = dimethyl formamide, HMPA = hexamethylphosphoramide, $\mathrm{Ms}=$ methylsufonate, $\mathrm{p}^{-\mathrm{TsOH}}=$ para-toluenesulfonic acid, 
NaHMDS = sodium hexame-thyldisilazide, TBAF $=$ tetra- $n$-butylammonium fluoride, TBS $=$ tert-butyldimethylsilyl, THF $=$ tetrahydrofuran, $\mathrm{Ts}=$ tosyl .

Initial attempts to close the central $D$ ring via an intramolecular Mitsunobu reaction or selective activation of the allylic alcohol with an activating reagent, such as tosyl chloride or triflic anhydride proved unsuccessful. In both cases the challenge of closing the seven membered ring was evident, as the products observed were those resulting from elimination of the activated alcohol or an exogenous nucleophile reacting with the activated leaving group (results not shown). We were encouraged to find that reaction of diol $\mathbf{5}$ with methylsufonate anhydride at low temperatures provided, as the sole product, the allylic mesylate 11 (Scheme 1c). This intermediate was unstable to purification, and attempts at acid or base mediated intramolecular etherification of this mesylate proved unsuccessful (Scheme 1c, $\mathbf{1 1} \rightarrow \mathbf{1 2}$ ). However, it was discovered that simply heating the reaction mixture once mesylation of diol $\mathbf{5}$ was complete led to the formation of the desired product $\mathbf{1 2}$ as the major product. Importantly, the desired trans stereochemistry across the $F G$ ring junction was observed, which had proven a significant challenge to access via other attempted methods (results not shown). The structure of triad $\mathbf{1 2}$ was confirmed by x-ray crystallography. Though encouraged by this result, the yield for this transformation was low (7\% over two steps), and thus it was decided to pursue a reductive etherification approach where formation of the presumed problematic reactive allylic carbocation could be avoided.

The use of reductive etherification strategies is well prece-dented in the MLP literature, however the stereospecificity of the reduction is highly substrate specific, and is known to vary based on the ring size and substitution pattern. Research by the Nicolaou group has revealed that intramolecular etherifications to form saturated 6,7 or 7,7 fused ring systems furnish a mixture of diasteromers. ${ }^{24}$ Though a similar approach had never been reported towards a 6,7 DHP-THO system, we were encouraged by precedence from the Tong group illustrating that enones were competent electrophiles for reductive etherifications. ${ }^{25}$ Desiring to explore the reductive etherification conditions on an intermediate that could readily be elaborated to include the $E F G$ dienal side-chain, we pursued synthesis of enone $\mathbf{1 3}$ with a pivoyl protected ethyl alcohol (Scheme 2). ${ }^{26}$

Drawing upon our previous synthesis, we modified the initial Ferrier reaction, adding this two-carbon segment in the form of 2-(trimethylsilyl)acetate. Saponification was followed by global silyl protection. Next, the sidechain was reduced and pivolyl protected to provide intermediate 14. Selective deprotection of the primary alcohol and its subsequent tosylation was followed by a Finkelstein reaction. Displacement of the formed iodide with triphenylphosphine yielded Wittig coupling partner 15. The Wittig coupling of 15 with TBS protected aldehyde $\mathbf{6}$ proceeded in modest yield with excellent $Z$ :E selectivity. Global deprotection provided diol 16. Previous work with diol 5 indicated that selective oxidation of the secondary allylic alcohol over the secondary aliphatic alcohol was achievable with DDQ (results not shown). Unfortunately, however, this reaction did not translate well to diol $\mathbf{1 6}$ with the side-chain in place. The transformation to $\mathbf{1 3}$ occurred in only $30 \%$ yield and multiple side-products were observed, including the desired enone with racemization occurring at the allylic carbon of the side-chain. Other oxidation conditions attempted $\left(\mathrm{MnO}_{2}, \mathrm{DMP}, \mathrm{Cu}(\mathrm{ACN})_{4} \mathrm{OTf} / \mathrm{NMI} / \mathrm{TEMPO}\right)$ were also unsuccessful. 
Reagents and conditions: a) TMSOTf, ethyl 2-(trimethylsilyl)acetate, $\mathrm{CH}_{2} \mathrm{Cl}_{2}, 2 \mathrm{~h},-10{ }^{\circ} \mathrm{C}$, $62 \%, 87: 13 \mathrm{dr}$; b) $\mathrm{K}_{2} \mathrm{CO}_{3}$, EtOH; then TBSCl, imid., DMF, 58\% (over 2 steps); c) LAH, THF, $0{ }^{\circ} \mathrm{C}$; then PivCl, pyr., $\mathrm{CH}_{2} \mathrm{Cl}_{2}, 69 \%$ (over 2 steps); d) $p$-TsOH, $\mathrm{MeOH}, 70 \%$; e) TsCl, 1:2 pyr. $/ \mathrm{CH}_{2} \mathrm{Cl}_{2}, 99 \%$; f) NaI, 2-butanone, $100{ }^{\circ} \mathrm{C}, 4 \mathrm{~d}, 50 \%$ (70\% brsm); g) $\mathrm{PPh}_{3}, 100{ }^{\circ} \mathrm{C}$, $24 \mathrm{~h}, 86 \%$; h) NaHMDS, then 6, 3:1 THF/HMPA, -78 to $-40{ }^{\circ} \mathrm{C}, 30 \mathrm{~min} ., 65 \%$, >95:5 Z/E; i) TBAF, THF, $88 \%$; j) DDQ, 1,4-dioxane $/ \mathrm{CH}_{2} \mathrm{Cl}_{2}, 80{ }^{\circ} \mathrm{C}, 30 \%$. DDQ = 2,3-dichloro-5,6dicyano-1,4-benzoquinone, HMPA = hexamethylphosphoramide, $\mathrm{LAH}=$ lithium aluminum hydride, Piv = pivaloyl, Ts = tosyl, TMS = trimethylsilyl.

Recognizing that this problem could be solved with an orthogonal protecting group strategy, PMB protected aldehyde $\mathbf{1 7}$ was rapidly accessed following a similar procedure to that previously detailed for TBS aldehyde $\mathbf{6}$ (Scheme 3). Coupling of PMB protected aldehyde 17 with phosphonium salt 15 provided the coupled product 18 in good (80\%) yield and excellent $Z / E$ selectivity. Desilylation followed by buffered oxidation provided the desired enone $\mathbf{1 9}$ as the sole product with no observable racemization at the remaining allylic center. To avoid competitive reduction, the benzylidene acetal protection group needed to be removed prior to reductive cyclization, ${ }^{27}$ and this was achieved with catalytic $p$-toluene sulfonic acid. We were pleased to find that reductive cyclization of intermediate $\mathbf{2 0}$ proceeded in 58\% yield to furnish $\mathbf{2 1}$ as exclusively the trans isomer. It is worth noting that, to our knowledge, this is the first example of a reductive cyclization to form a 7,6 ring system with in situ PMB deprotection. This important $E F G$ triad intermediate was accessed in 16 steps (LLS), and the stereochemistry at the $F G$ ring junction was confirmed by nOe NMR studies.

Reagents and conditions: a) NaHMDS then $17,3: 1$ THF/HMPA, -78 to $-40{ }^{\circ} \mathrm{C}, 30 \mathrm{~min}$., $80 \%$, >95:5 Z/E; b) TBAF, THF, $94 \%$; c) DMP, $\mathrm{NaHCO}_{3}, 0{ }^{\circ} \mathrm{C}$ to rt, $66 \%$; d) $p$-TsOH, $\mathrm{MeOH}$, quant.; e) TESOTf, $\mathrm{CH}_{3} \mathrm{CN}_{/} \mathrm{Et}_{3} \mathrm{SiH},-15^{\circ} \mathrm{C}, 15 \mathrm{~min}$., 58\%. DDQ = 2,3dichloro-5,6-dicyano-1,4-benzoquinone, $\mathrm{DMP}=$ Dess-Martin periodinane, HMPA = hexamethylphosphoramide, TES $=$ triethylsilyl.

In conclusion, two approaches towards the 6,7,6 triad of the $E F G$ core of tamulamides A and B have been examined. Successful synthesis of this triad was first achieved via selective allylic mesylation followed by intramolecular etherification to form the $E F G$ ring system in $7 \%$ yield. Ultimately, intramolecular etherification proved to be a more robust strategy, providing this $E F G$ motif in a much improved $58 \%$ yield. Importantly, this methodology was tolerant of the functional handle required for side-chain elaboration and occurred with excellent stereospecificity, providing exclusively the desired trans stereochemistry at the $F G$ ring junction.

\section{Supplementary Material}

Refer to Web version on PubMed Central for supplementary material.

\section{ACKNOWLEDGMENTS}

The authors thank the NIGMS for financial support (GM72566). Many thanks to Dr. Eric A. Standley (MIT), Liam Kelley (MIT) and Li Li (MIT) for HRMS analysis. We are grateful to Dr. Bruce Adams (MIT) for assistance with 
NMR spectroscopy and Dr. Tony Siu (Merck), Dr. Matthew Katcher (MIT), Dr. Kurt W. Armbrust (MIT), Dr. Satapanawat Sittihan (MIT) and Dr. Tamara Halkina (MIT) for many helpful conversations. Finally, we are grateful to Dr. Rachel L. Beingessner (MIT) for insightful discussions and input during the preparation of this manuscript.

\section{REFERENCES}

(1) (a). Nicoloau KC; Frederick MO; Aversa RJ Angew. Chem. Int. Ed 2008, 47, 7182-7225. (b)Vilojevic I; Jamison TF Mar. Drugs 2010, 8, 763-809. [PubMed: 20411125]

(2). Truxal LT; Bourdelais AJ; Jacocks H; Abraham WM; Baden DG J. Nat. Prod 2010, 73, 536-540. [PubMed: 20218657]

(3). Nagai H; Murata M; Torigoe K; Satake M; Yasumoto TJ Org. Chem 1992, 57, 5448-5453.

(4). Ronzitti G; Callegari F; Malaguti C; Rossini GP Br. J. Cancer 2004, 90, 1100-1107. [PubMed: 14997213]

(5). Russell JB; Houlihan AJ FEMS Microbiology Reviews 2003, 27, 65-74. [PubMed: 12697342]

(6). Nakanishi K Toxicon 1985, 23, 473-479. [PubMed: 3895583]

(7). Coxon JM; Hartshorn MP; Swallow WH Aust. J. Chem 1973, 26, 2521-2526.

(8). Heffron TP; Simpson GL; Merino E; Jamison TF J. Org. Chem 2010, 75, 2681-2701. [PubMed: 20302314]

(9). Wang Z; Cui Y-T; Xu Z-B; Qu J J. Org. Chem 2008, 73, 2270-2274. [PubMed: 18288864]

(10). Vilojevic I; Jamison TF Science 2007, 317, 1189-1192. [PubMed: 17761875] (b)Byers JA; Jamison TF Proc. Natl. Acad. Sci. U.S.A 2013, 110, 16724-16729. [PubMed: 24046369]

(11). Mosseau JJ; Morten CJ; Jamison TF Chem. Eur. J 2013, 19, 10004-10016. [PubMed: 23775936]

(12). Byers JA; Jamison TF PNAS. 2013, 110, 16724-16729. [PubMed: 24046369]

(13). To our knowledge, the only known THO-DHP synthesis was en route to an analog of ciguatoxin CTX1B and possessed trans relation-ship of hydrogen substituents across the ether bond of the DHP ring. Please see: Kira K; Isobe M Tet. Lett 2000, 41, 5951-5955.

(14). Kleinke AS; Webb D; Jamison TF Tetrahedron 2012, 68, 6999-7018.

(15). Kobayashi S; Takashi Y; Komano K; Alizadeh BH; Kawada Y; Oishi T; Tanaka S; Ogasawara Y; Sasaki S; Hirama M Tetrahedron 2004, 60, 8375-8396.

(16). Furuata H; Hasegawa Y; Mori Y Org. Lett 2009, 11, 4382-4385. [PubMed: 19731936]

(17). McGeough CP; Strom AE; Jamison TF Org. Lett 2019, 21, 3606-3609. [PubMed: 31046296]

(18). Valentine JC; McDonald FE; Neiwert WA; Hardcastle KI J. Am. Chem. Soc 2005, 127, 45864587. [PubMed: 15796519]

(19). Sasaki M; Tsukano C; Tachibana K; Org. Lett 2002, 4, 1747-1750. [PubMed: 12000289]

(20). This three-step procedure was modified from: Nicolaou KC; Hwang C-K; Marron BE; DeFrees SA; Couladourous EA; Abe Y; Carroll PJ; Synder JP J. Am. Chem. Soc 1990, 112, 3040-3054.

(21). Finkelstein conditions adapted from: Ireland RE; Smith MG J. Am. Chem. Soc 1988, 110, 854860.

(22). Yields for the triphenylphosphonium salt were highest when the reaction was run neat in molten $\mathrm{PPh}_{3}$ Procedure adapted from: Dondoni AD; Marra A; Mizuno M; Giovannin PPJ. Org. Chem 2002, 67, 4186-4199. [PubMed: 12054954] 3

(23). Smith AB; Beauchamp TJ; Lamarche MJ; Kaufman MD; Qiu Y; Arimoto H; Jones DR; Kobayashi KJ Am. Chem. Soc 2000, 122, 8654-8664.

(24). Nicolaou KC; Hwang C-K; Nugiel DA J. Am. Chem. Soc 1989, 111, 4136-4137.

(25). Zhu L; Song L; Tong R Org. Lett 2012, 14, 5892-5895. [PubMed: 23163770]

(26). For an example of a successful elaboration of an ethanol side chain to a dienal ester please see Ghosh AK; Liu W. J. Org. Chem 1997, 62, 7908-7909. [PubMed: 11671887]

(27). Sittihan S; Jamison TF J. Am. Chem. Soc 2019, 141, 11239-11244. [PubMed: 31283211] 
(a)

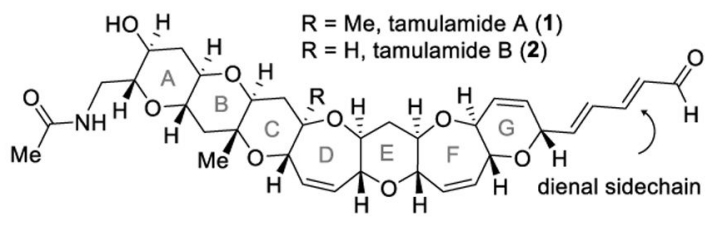

(c)

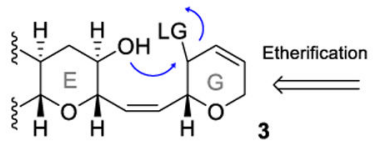

(b)

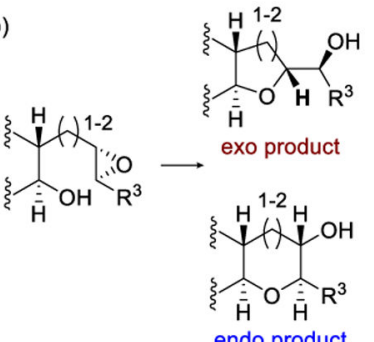

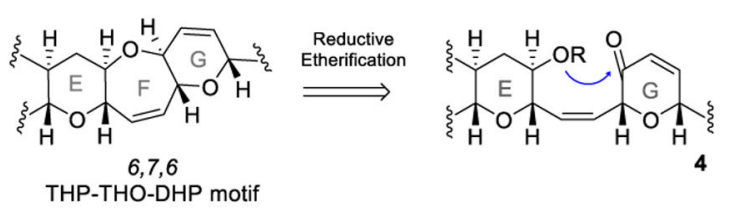

Figure 1.

(a) Tamulamides A (1) and B (2). (b) Exo and endo product formation. (c) Strategies to access the $E F G$ core.

Org Lett. Author manuscript; available in PMC 2020 October 04. 
(a)

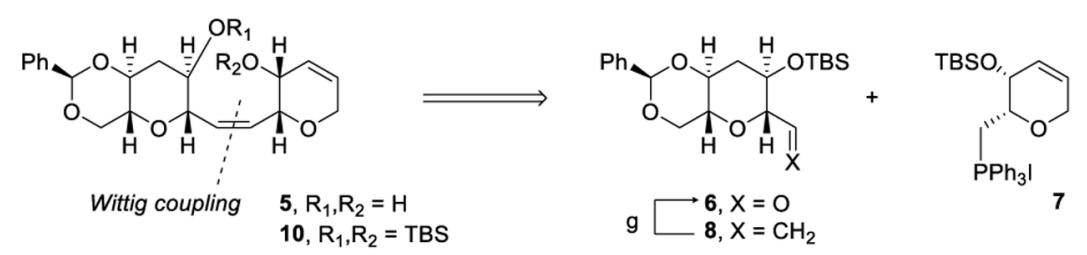

(b)

(c)
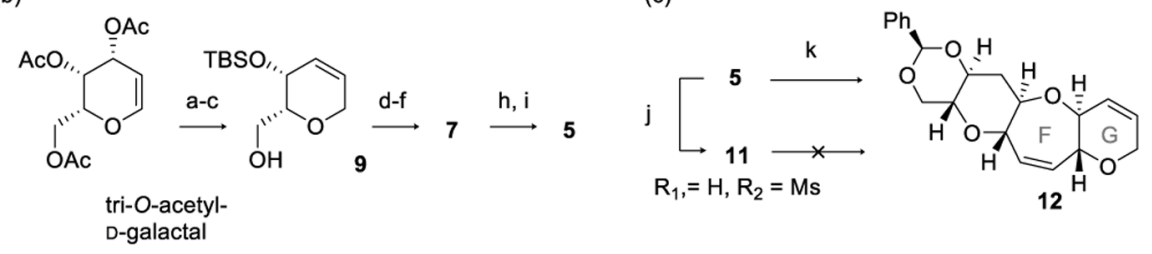

Scheme 1.

(a) Retrosynthetic analysis, (b) synthesis of and (c) initial results with intramolecular etherification precursor 5 . 

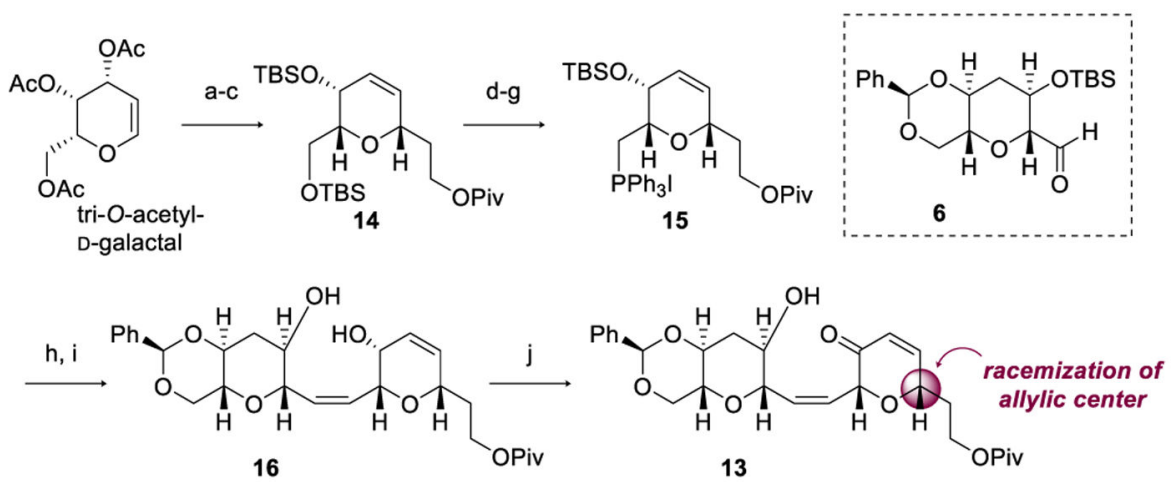

Scheme 2.

Synthesis of Wittig precursor $\mathbf{1 5}$ and problematic oxidation en route to reductive etherification precursor $\mathbf{1 3}$.

Org Lett. Author manuscript; available in PMC 2020 October 04. 


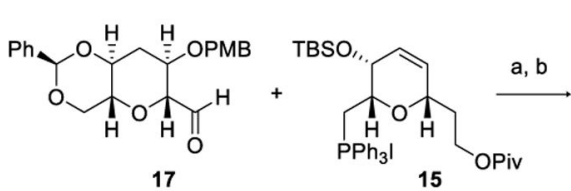

17

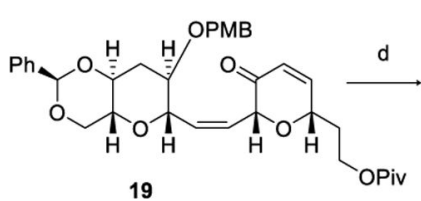

19

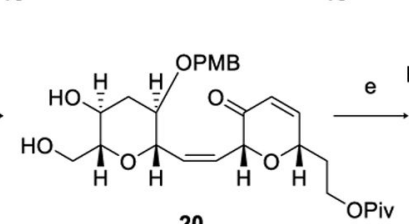

20
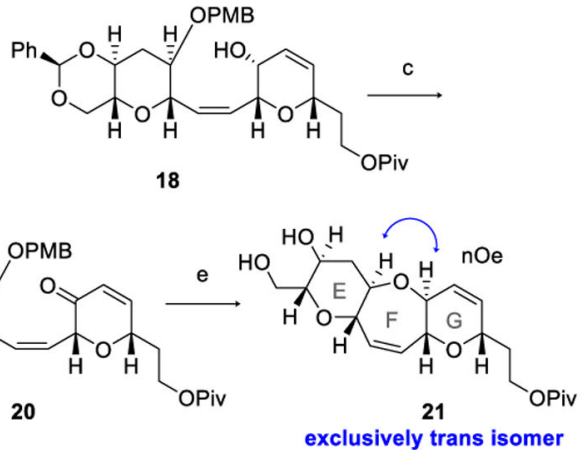

Scheme 3.

Synthesis of reductive etherification precursor $\mathbf{2 0}$ with side-chain handle in place, and successful formation of the $E F G$ triad 21. 